\title{
Efeito da utilização de gomas na viscosidade e nas características sensoriais de shake à base de farinha de banana verde
}

\author{
Effect of the use of gums on the viscosity and sensory characteristics of a shake based \\ on green banana flour
}

\section{Roberta Ribeiro Silva1*, Monique Assumpção', Patrícia Mello Fernandes'1, Tamires Almeida Feliciano", Camilla Ribeiro Vieira', Fernanda Laurides Ribeiro de Oliveira Lomeu' ${ }^{1}$}

1 Universidade Federal de Alfenas (UNIFAL), Departamento de Nutrição, Alfenas/MG - Brasil

\section{${ }^{*}$ Corresponding Author}

Roberta Ribeiro Silva, Universidade Federal de Alfenas (UNIFAL), Departamento de Nutrição, Rua Gabriel Monteiro da Silva, 700, Centro, CEP: 37130-000, Alfenas/MG - Brasil, e-mail: betaribeiro@hotmail.com

Cite as: Effect of the use of gums on the viscosity and sensory characteristics of a shake based on green banana flour. Braz. J. Food Technol., v. 21 , e2016052, 2018

\section{Resumo}

Este trabalho teve como objetivo testar diferentes proporções de gomas na elaboração de um shake desenvolvido com farinha de banana verde, além de avaliar as suas propriedades organolépticas. Analisaram-se seis formulações com os seguintes ingredientes: farinha de banana verde (FBV), leite em pó integral, sucralose, cacau e diferentes proporções de goma xantana (GX) e goma guar (GG), nas proporções (GX:GG): F1 (1:0); F2 (0:1); F3 (1:1); F4 (1:3); F5 (3:1); F6 sem estabilizante. As formulações de shake foram submetidas ao teste de viscosidade, realizado a cada três dias, durante um período de 15 dias. Foi realizada análise sensorial com voluntários, em cabines individuais. A aceitabilidade dos atributos aparência, aroma, sabor, textura e impressão global foi avaliada utilizando-se a escala hedônica de 9 pontos. Foram realizadas análises estatísticas utilizando-se o Teste de Variância (ANOVA) e o Teste de Tukey a 5\% de probabilidade. O estudo foi importante para demonstrar que a viscosidade dos produtos influencia nas características sensoriais e, dessa forma, interfere na escolha dos consumidores.

Palavras-chave: Emulsificantes; Goma xantana; Goma guar; Análise sensorial.

\section{Abstract}

This study aimed to test different proportions of gums and evaluate the organoleptic properties of a shake developed with green banana flour. Six formulations were analysed with the following ingredients: green banana flour (FBV), whole milk powder, sucralose, cocoa and different ratios of xanthan gum (GX) and guar gum (GG), in the proportions (GX:GG) of: F1 (1:0); F2 (0:1); F3 (1:1); F4 (1:3); F5 (3:1); F6 with no stabilizer. The viscosity of the samples was determined every three days for a period of 15 days. A sensory analysis was carried out with volunteers in individual booths. The acceptability of the attributes of appearance, aroma, flavour, texture and overall impression was evaluated using a 9-point hedonic scale. Statistical analyses were carried out using the variance test (ANOVA) and Tukey's test at 5\% probability. The study was important to demonstrate that the product viscosity influenced the sensory characteristics and hence interfered in the consumer choices.

Keywords: Emulsifiers; Xanthan gum; Guar gum; Sensory analysis.

\section{Introdução}

A escolha dos alimentos nas proporções adequadas, bem como a melhor forma de consumo, são aspectos importantes para o fornecimento de nutrientes. Entretanto, alguns alimentos, além da função de nutrir, agem trazendo algum outro benefício à saúde, conforme definido na Resolução n. ${ }^{\circ}$ 18/1999, da Agência Nacional de Vigilância Sanitária ANVISA (BRASIL, 1999). Dentre os alimentos e ingredientes com propriedades funcionais e/ou de saúde, os alimentos 
Efeito da utilização de gomas na viscosidade e nas características sensoriais de shake à base de farinha de banana verde

Silva, R. R. et al.

ricos em amido resistente vêm se destacando (BRASIL, 1999; NAVARRO et al., 2015).

A banana verde é um dos alimentos mais ricos em amido resistente. A banana está entre as frutas mais consumidas no mundo, sendo que o Brasil possui o maior consumo mundial per capita. Apresenta quantidades razoáveis de vitaminas $\mathrm{A}, \mathrm{B} 1$ e B2, pequenas quantidades de vitaminas $\mathrm{D}$ e $\mathrm{E}$, e maior percentagem de potássio, fósforo, cálcio e ferro do que a maçã ou a laranja. Sua alta concentração de amido possibilita seu processamento industrial em farinha, sendo, portanto, um produto de grande interesse como fonte alimentar (BORGES et al., 2009; FASOLIN et al., 2007).

De acordo com a literatura, a adição de farinha de banana verde (FBV) nos produtos, como substituta parcial da farinha de trigo, enriquece a preparação em relação aos alimentos tradicionais, elevando o valor nutricional sem alterar significativamente suas propriedades físicas e características sensoriais (FASOLIN et al., 2007). Assim, a versatilidade da FBV permite que seja incorporada em diversos alimentos, como, por exemplo, em bebidas lácteas tipo shake.

No desenvolvimento de produtos, é imprescindível otimizar parâmetros, como forma, cor, aparência, odor, sabor, textura e consistência, e buscar a interação dos diferentes componentes, com a finalidade de alcançar um equilíbrio integral que se traduza em uma qualidade excelente e que seja de boa aceitabilidade (BARBOZA et al., 2003).

Um dos maiores desafios enfrentados pela indústria de alimentos é manter a integridade dos seus produtos por períodos mais longos e assegurar que estes mantenham suas propriedades características e atratividade, até o momento do consumo. Neste cenário, substâncias capazes de assegurar as características físicas desses alimentos, como os estabilizantes, são utilizadas de maneira mais intensa (SOUTO et al., 2012).

Os estabilizantes são amplamente utilizados em indústrias alimentícias com esta finalidade. Define-se estabilizante como uma substância que torna possível a manutenção de uma dispersão uniforme de duas ou mais substâncias imiscíveis em um alimento, ou seja, evita que, com o tempo, os ingredientes se separem em diferentes fases; atua, dessa forma, promovendo uma interação homogênea de ingredientes. Os estabilizantes também auxiliam no aumento da viscosidade dos ingredientes e ajudam a evitar a formação de cristais que afetariam a textura do produto. São exemplos destes aditivos, as gomas xantana e guar (BRASIL, 1997; DEMIRCI et al., 2014).

A goma xantana é um polissacarídeo de extrema importância comercial. Esse polímero tem sido o mais utilizado em alimentos no Brasil e no mundo, como espessante/estabilizante. Foi aprovado pelo Food and Drug Administration (FDA) em 1969, sendo aplicado em inúmeros produtos de diferentes segmentos industriais, entre os quais alimentos, fármacos, cosméticos, químico e petroquímico, o que se deve principalmente a suas propriedades reológicas, que permitem a formação de soluções viscosas a baixas concentrações $(0,05$ a 1,0\%) e a estabilidade, em ampla faixa de $\mathrm{pH}$ e temperatura (LUVIELMO; SCAMPARINI, 2009).

A goma guar também pode ser empregada em bebidas como estabilizante ou, ainda, em sorvetes, molhos, misturas para bolo, como espessante (MUDGIL et al., 2014). Sua principal propriedade é a capacidade de hidratar-se rapidamente em água fria e atingir alta viscosidade (HUANG et al., 2006). Além disso, o baixo custo da goma guar é uma grande vantagem para sua utilização (OLIVEIRA et al., 2015).

Este trabalho teve como objetivo analisar o efeito das gomas xantana e guar na viscosidade e nas características sensoriais de um shake desenvolvido com farinha de banana verde.

\section{Material e métodos}

\subsection{Formulação do produto}

O shake foi composto pelos seguintes ingredientes: FBV, leite em pó integral, cacau, sucralose, goma xantana e/ou goma guar. Os ingredientes foram adquiridos em lojas de produtos naturais e farmácias de manipulação. As formulações-teste foram derivadas de uma composição-base, citada a seguir: $30 \mathrm{~g}$ de FBV; $20 \mathrm{~g}$ de leite em pó integral; $3 \mathrm{~g}$ de cacau em pó; 0,8 $\mathrm{g}$ de sucralose, e 0,3 g de goma xantana (LOMEU, 2015). A Tabela 1 apresenta a composição centesimal dos ingredientes da fórmula-base. A quantidade de goma xantana, presente na fórmula-base, foi considerada como $100 \%$ e, a partir desta quantidade, foram originadas as variações das fórmulas analisadas.

O produto em pó produzido foi acondicionado dentro de sacos plásticos individualmente ( $54,1 \mathrm{~g}$ cada), colocados em refrigerador doméstico, protegidos da

Tabela 1. Composição dos ingredientes por 100 gramas de parte comestível.

\begin{tabular}{lcccrr}
\multicolumn{1}{c}{ Ingredientes } & Calorias (kcal) & Proteínas (g) & Gorduras totais & Carboidrato (g) & Fibras \\
FBV & 310,00 & 4,50 & 3,50 & 65,00 & 11,00 \\
Leite em pó integral & 496,15 & 25,00 & 26,92 & 38,46 & 0,00 \\
Cacau em pó & 290,00 & 5,00 & 10,00 & 45,00 & 10,00 \\
Sucralose & 416,60 & 0,00 & 0,00 & 0,00 & 83,30 \\
Goma Xantana & 330,00 & 10,00 & 0,00 & 80,00 & 76,00 \\
\hline
\end{tabular}


Efeito da utilização de gomas na viscosidade e nas características sensoriais de shake à base de farinha de banana verde

Silva, R. R. et al.

luz por sacos pretos durante todo o período, sendo que cada saco era reconstituído com $150 \mathrm{~mL}$ de água mineral à temperatura ambiente $\left(26,8 \pm 2,0^{\circ} \mathrm{C}\right)$, nos momentos das análises.

Foram desenvolvidas e testadas diferentes proporções entre as gomas isoladas e combinadas, com quantidade fixa dos demais ingredientes. A formulação 1 (F1) era composta por 0,3 g de goma xantana; a formulação 2 (F2) continha 0,3 g de goma guar; a formulação 3 (F3) apresentava 0,15 g de goma xantana e 0,15 g de goma guar; a formulação 4 (F4) com 0,075 g goma xantana e 0,225 g goma guar; a formulação 5 (F5) com 0,225 g goma xantana e 0,075 g goma guar, e a formulação 6 (F6) não apresentava nenhuma goma, sendo utilizada como controle.

As formulações desenvolvidas foram realizadas no Laboratório de Técnica Dietética da Faculdade de Nutrição (FANUT) da Universidade Federal de Alfenas - UNIFAL-MG.

\subsection{Análise da viscosidade}

Para a determinação da viscosidade das formulações analisadas (1 a 6), utilizou-se um viscosímetro da marca Brookfield (Modelo RVT), disponível no Laboratório de Técnica Dietética da FANUT da UNIFAL-MG, sendo que o instrumento é equipado com cilindros de diâmetros diferentes (spindles). Cada spindle é apropriado para medir a viscosidade de fluidos em uma faixa específica: os de menor diâmetro são utilizados para determinar as maiores viscosidades; e os de maior diâmetro, as menores viscosidades.

A metodologia para utilização do aparelho baseou-se no manual de instruções Brookfield Dial Viscometer (BROOKFIELD, 2005). O primeiro ensaio realizado foi a determinação da faixa ideal de velocidade de rotação do spindle, para as amostras. Além da faixa de velocidade, foram testadas as combinações entre spindles e recipientes para os testes disponíveis. Dentro destas especificações, ficou determinado o spindle adequado (s 63), para as formulações analisadas, bem como o recipiente ideal (copos descartáveis $200 \mathrm{~mL}$ ) para receber o conteúdo da amostra (aproximadamente $150 \mathrm{~mL}$ ), já que o raio do copo influencia no torque. Durante o ensaio, as amostras foram submetidas à velocidade de 30 rpm (rotações por minuto) e sua viscosidade foi medida três vezes, uma a cada intervalo de 15 segundos. Cabe ressaltar que, no viscosímetro Brookfield, as leituras são corrigidas por um fator apresentado no manual do equipamento; no caso, o fator de multiplicação para o spindle 63, na velocidade de 30 rpm, foi de 40, obtendo-se os resultados na unidade mPa.s (milipascal segundo).

Foram realizadas análises da viscosidade em cada variação de fórmula (Formulação 1 a 6) do produto. As análises foram realizadas em seis momentos $\left(t_{0} ; t_{1} ; t_{2} ; t_{3} ; t_{4}, e t_{5}\right)$, com intervalos de três dias, totalizando um período de 15 dias, sendo este experimento de análise realizado em triplicata.

\subsection{Análise do pH}

$\mathrm{O} \mathrm{pH}$ de cada uma das formulações de shake (F1, F2, F3, F4, F5 e F6) foi avaliado de acordo com o método do Instituto Adolfo Lutz (IAL, 2008), com o uso de pHmetro MPA-210 (MS TECNOPON Equipamentos Especiais Ltda., Piracicaba, SP) previamente calibrado em soluções-tampão padrão com pH 7,0 e pH 4,0. Após a calibração do aparelho, foram transferidos $20 \mathrm{~mL}$ de cada formulação para um béquer de $25 \mathrm{~mL}$, sendo realizada a imersão do eletrodo, seguida da leitura. As análises foram feitas nas bebidas reconstituídas em água à temperatura ambiente $\left(26,8 \pm 2,0^{\circ} \mathrm{C}\right)$, em triplicata.

\subsection{Avaliação sensorial e de aceitabilidade}

A avaliação sensorial foi realizada com voluntários não treinados, da comunidade acadêmica da UNIFAL-MG, no Laboratório de Avaliação Sensorial da FANUT, em cabines individuais, em que o público alvo foram consumidores com idade acima de 18 anos.

Os provadores foram esclarecidos de forma completa e em linguagem leiga a respeito do protocolo de pesquisa. O consentimento para participar do estudo foi comprovado com a assinatura de um Termo de Consentimento Livre e Esclarecido, que foi aprovado pelo Comitê de Ética em Pesquisa da UNIFAL-MG, n. ${ }^{\circ}$ 25496313.8.0000.5142.

As seis formulações desenvolvidas foram servidas aleatoriamente aos julgadores, em copos plásticos, com quantidades padronizadas em $50 \mathrm{~mL}$, codificadas com números aleatórios de três dígitos, sendo oferecidos $150 \mathrm{~mL}$ de água para limpeza da cavidade oral entre as avaliações das amostras.

A aceitação das amostras foi avaliada através dos atributos aroma, aparência, sabor, textura e impressão global, utilizando-se escala hedônica de 9 pontos, cujos extremos correspondem a 'desgostei muitíssimo' (1) e 'gostei muitíssimo' (9) (REIS; MINIM, 2006).

\subsection{Análise estatística}

Para comparar as médias de aceitação sensorial das formulações, em função do nível de substituição dos ingredientes, e também para analisar quais formulações mantiveram-se estáveis ao longo do tempo, com a análise de viscosidade, foram realizados a Análise de Variância (ANOVA) e o Teste de Tukey, com nível de significância de $5 \%$. 
Efeito da utilização de gomas na viscosidade e nas características sensoriais de shake à base de farinha de banana verde

Silva, R. R. et al.

\section{Resultados e discussão}

\subsection{Análise da viscosidade}

De acordo com os resultados da Tabela 2, observou-se que os valores de viscosidade inicialmente medidos (to) diminuíram ao longo do tempo de armazenamento de 15 dias (t15). Segundo Arana (2012), a diminuição da viscosidade, ao longo do tempo, deve-se ao colapso progressivo de forças que mantêm as partículas em suspensão, o que resulta na redução do tamanho de partículas estruturais, oferecendo menor resistência.

Porém, é importante ressaltar que, em parte das amostras, a viscosidade aumentou no tempo t1. Isto ocorreu porque, em t0, as amostras se encontravam em temperatura ambiente, visto que a análise foi realizada logo após a formulação do produto. Em t1, as amostras tinham sido estocadas em temperatura de refrigeração. De acordo com Pagno et al. (2014), a temperatura influencia na viscosidade das bebidas, obtendo-se geralmente uma viscosidade mais elevada em temperaturas mais baixas.

As formulações F3 e F5 apresentaram menor perda de viscosidade ao longo do tempo, fato que pode ser relacionado com a associação da goma guar à goma xantana, cujo efeito tende a promover um aumento na viscosidade de soluções. Além disso, segundo Maruyama et al. (2006), maiores valores de viscosidade aparente são reportados em soluções contendo goma xantana; assim, a maior viscosidade obtida pelas formulações F1, F3 e F5, no tempo 5 (t5), ocorreu conforme seria esperado.

Ospina et al. (2012) avaliaram a ação das gomas xantana e guar em um leite aromatizado com cacau e observaram que a melhor combinação, para manutenção da estabilidade, foi obtida com $70 \%$ de xantana e 30\% de guar; nessa proporção, não foi observada a separação de componentes e a sedimentação do cacau foi mínima, demonstrando a sinergia entre as gomas.

A amostra $F_{6}$, cuja formulação não continha nenhum tipo de estabilizante, apresentou menor viscosidade em todas as épocas de análise, indicando a importância do uso de estabilizantes para manter as características organolépticas do produto.
Deshpande et al. (2005) desenvolveram uma bebida de soja com sabor de chocolate e amendoim (adicionado de carragena e lecitina de soja) e encontraram valores de viscosidade em um intervalo de 17,7 a 131,8 mPa.s. Abedi et al. (2012) encontraram o valor de $165 \pm 0,40 \mathrm{mPa}$.s para a viscosidade de uma bebida láctea adicionada de framboesa, contendo $0,35 \%$ de pectina. Os resultados encontrados na literatura, citados anteriormente, para a viscosidade de bebidas, são semelhantes aos resultados verificados na última época de análise (t5) das formulações de shake no presente estudo, ou seja, após 15 dias de produção.

A análise da viscosidade aparente é um parâmetro reológico de simples execução, cujo resultado pode ser correlacionado com a avaliação sensorial, tornando-se indispensável.

\subsection{Análise do pH}

Observou-se que as formulações estudadas apresentaram pH entre 6,18 e 6,225 (Tabela 3). Provavelmente, essas diferenças são consequência das diferentes proporções de gomas utilizadas em cada uma das formulações.

A ação da goma xantana abrange uma extensa faixa de $\mathrm{pH}$, de 1 a 13, estando o shake em estudo na faixa de boa atuação da goma. A goma guar é influenciada por soluções com baixo $\mathrm{pH}$, sendo efetiva em produtos ácidos. Segundo Nikaedo et al. (2004), a goma guar confere textura macia e reduz a sinérese em produtos de laticínios. Portanto, verificou-se que o pH das soluções foi favorável para a atuação dos estabilizantes.

\subsection{Avaliação sensorial e de aceitabilidade}

Participaram da análise sensorial (Tabela 4) 109 provadores, os quais avaliaram os seguintes atributos: aparência, aroma, sabor, textura e impressão global. Para o atributo aparência, verificou-se que as formulações $F_{1}, F_{2}$ e $F_{5}$ apresentam melhor aceitação, além de não haver diferença estatisticamente significante entre estas.

Tabela 2. Viscosidade (mPa.s) das formulações de shake com diferentes concentrações de estabilizantes.

\begin{tabular}{|c|c|c|c|c|c|c|}
\hline $\begin{array}{c}\text { Tempo / } \\
\text { Formulações }\end{array}$ & F1 & F2 & F3 & F4 & F5 & F6 \\
\hline to & $370,00^{b}$ & $313,00^{a}$ & $323,00^{\mathrm{ac}}$ & $409,00^{a}$ & $264,00^{\text {ac }}$ & $60,00^{a}$ \\
\hline $\mathrm{t} 1$ & $512,00^{a}$ & $286,25^{a}$ & $375,58^{a c}$ & $470,20^{a}$ & $413,50^{\mathrm{ac}}$ & $59,23^{a}$ \\
\hline t2 & $341,28^{b}$ & $198,00^{a b}$ & $414,00^{a}$ & $393,23^{a}$ & $438,53^{a}$ & $68,45^{a}$ \\
\hline t3 & $185,18^{c}$ & $67,58^{b}$ & $245,90^{b c}$ & $194,52^{b}$ & $213,53^{b c}$ & $49,98^{a}$ \\
\hline t4 & $149,18^{c}$ & $76,30^{b}$ & $313,83^{a c}$ & $151,07^{b c}$ & $250,08^{a c}$ & $41,73^{a}$ \\
\hline t5 & $147,45^{c}$ & $38,50^{b}$ & $190,48^{b c}$ & $75,45^{c}$ & $192,03^{b c}$ & $3,33^{b}$ \\
\hline
\end{tabular}

Médias identificadas com letras iguais, em uma mesma coluna, não diferem significativamente entre si, para $p<0,05$. t: análise da viscosidade nos tempos de zero a 5 dias. F1: $100 \%$ de goma xantana; F2: 100\% de goma guar; F3: 50\% goma xantana e 50\% goma guar; F4: 25\% goma xantana e 75\% goma guar; F5: 75\% goma xantana e 25\% goma guar; F6: Controle, sem gomas. 
Efeito da utilização de gomas na viscosidade e nas características sensoriais de shake à base de farinha de banana verde

Silva, R. R. et al.

Tabela 3. Valores do pH e da viscosidade (mPa.s) nas formulações de shake logo após o preparo.

\begin{tabular}{|c|c|c|c|}
\hline Formulações & Viscosidade (mPa.s) & pH & $\mathbf{P}$ \\
\hline F1 & $360,0 \pm 0,0$ & $6,18^{a} \pm 0,000$ & \multirow{6}{*}{0,007} \\
\hline F2 & $302,0^{b} \pm 2,83$ & $6,22^{b} \pm 1,410$ & \\
\hline F3 & $318,0^{b} \pm 2,83$ & $6,195^{\mathrm{abc}} \pm 0,875$ & \\
\hline F4 & $406,0 \pm 8,49$ & $6,22^{b c} \pm 0,000$ & \\
\hline F5 & $252,0 \pm 16,97$ & $6,215^{b c} \pm 0,855$ & \\
\hline F6 & $60,0 \pm 0,0$ & $6,255 \pm 0,815$ & \\
\hline
\end{tabular}

Dados expressos como a média de duas medidas \pm desvio padrão; médias marcadas com letras iguais, em uma mesma coluna, não diferem significativamente entre si. F1: 100\% de goma xantana; F2: 100\% de goma guar; F3: 50\% goma xantana e 50\% goma guar; F4: 25\% goma xantana e $75 \%$ goma guar; F5: $75 \%$ goma xantana e $25 \%$ goma guar; F6: Controle, sem gomas.

Tabela 4. Teste sensorial afetivo para as formulações de shake em diferentes concentrações de estabilizantes.

\begin{tabular}{cccccc} 
Formulações & Aparência & Aroma & Sabor & Textura & Impressão Global \\
\hline F1 & $7,50^{\mathrm{a}}$ & $6,50^{\mathrm{a}}$ & $5,83^{\mathrm{ab}}$ & $6,35^{\mathrm{abc}}$ & $6,32^{\mathrm{a}}$ \\
F2 & $7,28^{\mathrm{a}}$ & $6,54^{\mathrm{a}}$ & $6,08^{\mathrm{a}}$ & $6,61^{\mathrm{a}}$ & $6,52^{\mathrm{a}}$ \\
F3 & $6,81^{\mathrm{b}}$ & $6,49^{\mathrm{a}}$ & $5,85^{\mathrm{ab}}$ & $6,20^{\mathrm{abc}}$ & $6,30^{\mathrm{a}}$ \\
F4 & $6,72^{\mathrm{b}}$ & $6,44^{\mathrm{a}}$ & $5,67^{\mathrm{ab}}$ & $6,00^{\mathrm{bc}}$ & $6,15^{\mathrm{a}}$ \\
F5 & $7,28^{\mathrm{a}}$ & $6,39^{\mathrm{a}}$ & $5,76^{\mathrm{ab}}$ & $6,49^{\mathrm{ab}}$ & $6,34^{\mathrm{a}}$ \\
F6 & $6,67^{\mathrm{b}}$ & $6,22^{\mathrm{a}}$ & $5,38^{\mathrm{b}}$ & $5,85^{\mathrm{c}}$ & $5,68^{\mathrm{b}}$ \\
\hline
\end{tabular}

Médias marcadas com letras iguais, em uma mesma coluna, não diferem significativamente entre si. F1: 100\% de goma xantana; F2: 100\% de goma guar; F3: 50\% goma xantana e 50\% goma guar; F4: 25\% goma xantana e 75\% goma guar; F5: 75\% goma xantana e 25\% goma guar; F6: Controle, sem gomas. Análise realizada com as formulações em to, ou seja, logo após o preparo das formulações.

Apesar de não ter ocorrido diferença estatística entre as formulações, a amostra $F_{2}$ apresentou a maior média para os seguintes quesitos: aroma, sabor, textura e impressão global. Portanto, formulações com goma guar isolada $\left(F_{2}\right)$ apresentaram menores valores para viscosidade e melhor aceitação geral nos parâmetros avaliados. Assim, observou-se que a viscosidade elevada é indesejável entre os consumidores. Jadhav et al. (2003) estudaram a aceitação de um milk shake de sapoti com diferentes concentrações (10, 20 e 30\%) de polpa e perceberam que o aumento da viscosidade não agradava os provadores. Santos et al. (2008), em sua análise da influência da concentração de soro na aceitação sensorial de bebida láctea fermentada com polpa de manga, identificou que a formulação que apresentou consistência mais firme (mais viscosa) foi a de menor aceitabilidade em relação às de consistência intermediária.

As formulações de shake F1, F2, F3, e F5 apresentaram médias de aceitação $\geq 7,0$, na impressão global. Segundo Teixeira et al. (1987), para que o produto seja considerado aceito por suas propriedades sensoriais, é importante que obtenha um índice de aceitabilidade de, no mínimo, $70 \%$. Assim, pode-se dizer que as bebidas apresentam potencial de mercado, o qual está em crescente expansão, pois os consumidores estão buscando cada vez mais por produtos que aliem saúde, sabor e praticidade.

Destaca-se ainda que a formulação F6 apresentou a menor média em todos os quesitos, sendo que, na impressão global, apresentou diferença estatística significante em relação às demais amostras. Pode-se concluir, com este resultado, como o uso de estabilizantes é importante para manter as características sensoriais dos produtos.

\section{Conclusão}

Conclui-se que as diferentes proporções de gomas guar e xantana, empregadas na produção do shake à base de farinha de banana verde, influenciaram na viscosidade e aceitação do produto, que afetam as características sensoriais e, dessa forma, interferem na escolha dos consumidores.

\section{Agradecimentos}

Agradecimentos pela oportunidade de pesquisa e pela bolsa concedida ao PIBIC/CNPQ da Universidade Federal de Alfenas, Minas Gerais, Brasil.

\section{Referências}

ABEDI, F.; SANI, A. M.; KARAZHIYAN, H. Effect of some hydrocolloids blend on viscosity and sensory properties of raspberry juice-milk. Índia: Association of Food Scientists \& Technologists, 2012. 5 p.

ARANA, I. Physical properties of foods: novel measurement techniques and applications. Boca Raton: CRC Press, 2012. 163 p. http://dx.doi.org/10.1201/b11542.

BARBOZA, L. M. V.; FREITAS, R. J. S.; WASZCZYNSKYJ, N. Desenvolvimento de produtos e análise sensorial. Revista Brasileira de Alimentos, n. 18, p. 34-35, 2003.

BORGES, A. M.; PEREIRA, J.; LUCENA, E. M. P. Caracterização de farinha de banana verde. Ciência e Tecnologia de Alimentos, 
Efeito da utilização de gomas na viscosidade e nas características sensoriais de shake à base de farinha de banana verde

Silva, R. R. et al.

v. 29, n. 2, p. 333-339, 2009. http://dx.doi.org/10.1590/S010120612009000200015

BRASIL. Portaria n 540, de 27 de outubro de 1997. Aprova o regulamento técnico: aditivos alimentares: definições, classificação e emprego. Diário Oficial [da] República Federativa do Brasil, Brasília, DF, 28 out. 1997.

BRASIL. Agência Nacional de Vigilância Sanitária. Resolução n 18, de 30 de abril de 1999. Aprova o Regulamento Técnico que estabelece as diretrizes básicas para análise e comprovação de propriedades funcionais e ou de saúde alegadas em rotulagem de alimentos, constante do anexo desta portaria. Diário Oficial [da] República Federativa do Brasil, Brasília, DF, 3 maio 1999.

BROOKFIELD ENGINEERING LABORATORIES - BROOKFIELD. Brookfield Dial Viscometer: operating instructions. Middleboro: Brookfield, 2005. 35 p. (Manual, M/85-150-P700).

DEMIRCI, Z. O.; YILMAZ, I.; DEMIRCI, A. S. Effects of xanthan, guar, carrageenan and locust bean gum addition on physical, chemical and sensory properties of meatballs. Journal of Food Science and Technology, v. 51, n. 5, p. 936-942, 2014. PMid:24803701. http://dx.doi.org/10.1007/s13197-011-0588-5.

DESHPANDE, R. P. M. S.; CHINNAN, M. S.; MCWATTERS, K. $H$. Nutritional, physical and sensory characteristics of various chocolate-flavored peanut soy beverage formulations. Journal of Sensory Studies, v. 20, n. 2, p. 130-146, 2005. http://dx. doi. org/10.1111/j.1745-459X.2005.00015.x.

FASOLIN, L. H.; ALMEIDA, G. C.; CASTANHO, P. S.; NETTOOLIVEIRA, E. R. Biscoitos produzidos com farinha de banana: avaliações química, física e sensorial. Ciência e Tecnologia de Alimentos, v. 27, n. 3, p. 524-529, 2007. http://dx.doi.org/10.1590/ S0101-20612007000300016.

HUANG, Y.; YU, H.; XIAO, C. Efects of Ca2+ crosslinking on structure and properties of waterborne polyurethane-carboxymethylated guar gum films. Carbohydrate Polymers, v. 66, n. 4, p. 500-513, 2006. http://dx.doi.org/10.1016/j.carbpol.2006.04.001.

INSTITUTO ADOLFO LUTZ - IAL. Métodos físico-químicos para análise de alimentos. 4. ed. São Paulo: IAL, 2008. 1020 p. Versão eletrônica.

JADHAV, V. S.; AWAZ, H. B.; PATIL, G. R.; THOMBRE, B. M. Studies on preparation of sapota milk shake. Journal of Maharashtra Agricultural Universities, v. 27, n. 3, p. 306-308, 2003.

LOMEU, F. L. R. O. Bebida láctea funcional tipo "shake" a base de farinha de banana (Musa spp.) verde: desenvolvimento, aceitabilidade e efeito no estado nutricional antropométrico, metabólico e dietético de mulheres com excesso de peso e adiposidade abdominal. 2015. 127 f. Dissertação (Mestrado em Biociências aplicadas à Saúde)-Faculdade de Nutrição, Universidade Federal de Alfenas, Alfenas, 2015.

LUVIELMO, M. M.; SCAMPARINI, A. R. P. Goma xantana: produção, recuperação, propriedades e aplicação. Estudos Tecnológicos, v. 5, n. 1, p. 50-67, 2009. http://dx.doi.org/10.4013/ete.2009.51.04.
MARUYAMA, L. Y.; CARDARELLI, H. R.; BURITI, F. C. A.; SAAD, S. M. I. Textura instrumental de queijo petit-suisse potencialmente probiótico: influência de diferentes combinações de gomas. Food Science and Technology, v. 26, n. 2, p. 386-393, 2006. http://dx.doi.org/10.1590/S0101-20612006000200022.

MUDGIL, D.; BARAK, S.; KHATKAR, B. S. Guar gum: processing, properties and food applications: a review. Journal of Food Science and Technology, v. 51, n. 3, p. 409-418, 2014. PMid:24587515. http://dx.doi.org/10.1007/s13197-011-0522-x.

NAVARRO, S. D.; MAURO, M. O.; PESARINI, J. R.; OGO, F. M.; OLIVEIRA, R. J. Resistant starch: a functional food that prevents DNA damage and chemical carcinogenesis. Genetics and Molecular Research, v. 14, n. 1, p. 1679-1691, 2015. PMid:25867310. http://dx.doi.org/10.4238/2015.March.6.14.

NIKAEDO, P. H. L.; AMARAL, F. F.; PENNA, A. L. B. Caracterização tecnológica de sobremesas lácteas achocolatadas cremosas elaboradas com concentrado protéico de soro e misturas de gomas carragena e guar. Revista Brasileira de Ciências Farmacêuticas, v. 40, n. 3, p. 397-404, 2004. http://dx.doi. org/10.1590/S1516-93322004000300016

OLIVEIRA, E. G.; CAMPOS, R. S.; MACHADO, A. S.; PEREIRA, J. F.; ARAÚJO, T. G. Avaliação da goma guar no desenvolvimento de comprimidos matriciais de liberação controlada de teofilina. Polímeros, v. 25, n. spe, p. 54-58, 2015. http://dx.doi. org/10.1590/0104-1428.1756.

OSPINA, M. M.; SEPULVEDA, J. U.; RESTREPO, D. A.; CABRERA, K. R.; SUÁREZ, H. Influencia de goma xantan y goma guar sobre lãs propiedades reológicas de lechesaborizadaconcocoa. Biotecnología en el Sector Agropecuario y Agroindustrial, v. 10, n. 1, p. 51-59, 2012

PAGNO, C. H.; SOUZA, L. F.; FLORES, S. H.; JONG, E. V. Desenvolvimento de espessante alimentar com valor nutricional agregado, destinado ao manejo da disfagia. Ciência Rural, v. 44, n. 4, p. 710-716, 2014. http://dx.doi.org/10.1590/S010384782014000400023.

REIS, R.; MINIM, V. P. R. Testes de aceitação. In: MINIM, V. P. R. Análise sensorial: estudos com consumidores. Viçosa: Editora da UFV, 2006.

SANTOS, C. T.; COSTA, A. R.; FONTAN, G. C. R.; FONTAN, R. C. I.; BONOMO, R. C. F. Influência da concentração de soro na aceitação sensorial de bebida láctea fermentada com polpa de manga. Alimentos e Nutrição, v. 19, n. 1, p. 55-60, 2008.

SOUTO, L. I. A.; SILVA, J. F.; FIGUEIREDO, R. A. R.; SILVA, G. $M$. Estabilizantes alimentares: efeitos desejáveis nos alimentos. In: SIMPÓSIO PARAIBANO EM SAÚDE: TECNOLOGIA, SAÚDE E MEIO AMBIENTE À SERVIÇO DA SAÚDE, 2012, Paraíba. Anais.. João Pessoa: Impressos Adilson, 2012. 218 p.

TEIXEIRA, E.; MEINERT, E. M.; BARBETTA, P. A. Análise sensorial de alimentos. Florianópolis: UFSC, 1987. 180 p. 